\title{
Amino acid metabolism and prognostic markers in ischemic stroke patients
}

chenxi zhang

Beijing Tiantan Hospital

lijuan wang

Beijing Tiantan Hospital

nan qiu

Beijing Tiantan Hospital

yijun shi

Beijing Tiantan Hospital

yaowei ding

Beijing Tiantan Hospital

guoge li

Beijing Tiantan Hospital

guanghui zheng

Beijing Tiantan Hospital

guojun zhang ( $\sim$ guojun.zhang@ccmu.edu.cn )

Beijing Tiantan Hospital https://orcid.org/0000-0003-4911-5784

\section{Research Article}

Keywords: Ischemic stroke, Amino acid, Metabolism, Prognosis

Posted Date: January 19th, 2022

DOI: https://doi.org/10.21203/rs.3.rs-1262523/v1

License: (c) (i) This work is licensed under a Creative Commons Attribution 4.0 International License.

Read Full License 


\section{Abstract}

Ischemic stroke continues to be a serious public health problem worldwide, we compared the amino acid profiles of convalescent patients with ischemic stroke with healthy subjects by High Performance Liquid Chromatography (HPLC), 48 convalescent patients with ischemic stroke and 48 healthy controls were recruited, finding that the levels of Phosphoserine(P-Ser), Glutamic Acid(Glu), Isoleucine(Ile), Leucine(Leu), Phenylalanine(Phe), Arginine(Arg), Proline(Pro) in stroke patients increased significantly, and the levels of Taurine(Tau), Cystine(Cys), Methionine(Met), b-Alanine(b-Ala), Histidine(His) lowered in patients compared to controls $(\mathrm{P}<0.05)$. Furthermore, it was particularly important to find indicators that could judge the prognosis of patients, the Tau/Glu level could be used as a potential prognosis marker and considering convalescent stroke patients have a good prognosis when the ratio $>0.79$ in our study.

\section{Introduction}

Stroke is a complex neurological syndrome, which is one of the most important causes of disability and the second most common cause of death worldwide(H. Wang et al., 2020). Most stroke patients will have different degrees of neurological, sensory and other dysfunctions after treatment, which seriously affects patients' daily life and work; Although the treatment of patients with stroke has improved greatly in recent years, it still has a high recurrence rate(Tu et al., 2017); Therefore, it is particularly important to investigate the metabolism and find some indicators that can evaluate the prognosis of stroke patients for the optimized care and allocation of healthcare resources.

Metabolomics, which focuses on the profiling of small metabolites in biofluids, cells and tissues, aiming to reveal the metabolic state of biological systems; metabolites are the substrates and products of metabolism that drive essential functions, such as energy production and storage, signal transduction and apoptosis, which can regulate or affect the environment in which they are produced (Johnson, Ivanisevic, \& Siuzdak, 2016; Johnson, Patterson, Idle, \& Gonzalez, 2012). Increase or decrease of the amino acid in the brain tissue have a toxic effect on the brain tissue, and it has been recognized as one of the mechanisms of stroke injury(Gomi, Karp, \& Greenberg, 2000; Ramasamy, Dutta, Kannan, \& Chandramouleeswaran, 2021). When a stroke occurs, brain tissue edema, inflammation or neuronal damage can also cause significant changes in serum amino acid metabolite group. Therefore, the levels

of free amino acids in serum could partially reflect the status of metabolic disorders, which may help to distinguish stroke patients with healthy subjects and evaluate the prognosis of stroke patients (Xie \& Li, 2015).

In this study, we compared the amino acid profiles of convalescent patients with ischemic stroke with healthy subjects by HPLC, aiming to find the metabolic differences between two groups. At the same time, we monitored the trend of amino acid changes continuously and tried to seek some prognosis biomarkers for ischemic stroke patients.

\section{Materials And Methods}




\subsection{Study design and methods}

Convalescent patients with ischemic stroke (after 14 days of the onset of ischemic stroke diagnostic) were recruited from Huguosi Hospital of TMC affiliated to Beijing University of Chinese Medicine. The study was approved by ethics committee, and informed consent was obtained from all individual participants included in the study. Stroke severity was assessed on admission using the National Institutes of Health Stroke Scale (NIHSS) by a neurologist, and the NIHSS scores of all patients were fluctuated between 5-15. Barthel index (BI) was used to assess the ability of daily living of these patients when they were discharged, and the patients were divided into a good prognosis group (BI>60) and a poor prognosis group $(\mathrm{BI} \leq 60)$. Healthy subjects (individuals without history of ischemic or hemorrhagic stroke) were included at the same time, which were considered as the control group. Those subjects had no statistical significance on basic characteristics, such as age\sex\other illnesses and so on, and the information of all subjects was listed in Table 1. Blood samples were collected according to entry criteria, and all the samples analysis were performed by HPLC using Amino Acid Analyzer (Hitachi L8900).

Table1 Information of all subjects in this study

\begin{tabular}{|l|lll|}
\hline \multicolumn{3}{|c|}{ Group } \\
\hline Information & Controls & Ischemic stroke patients \\
\hline Age rangelyearl & $39-75$ & $31-89$ \\
\hline Sex & & \\
\hline Female & 20 & 15 \\
\hline \multicolumn{1}{|c|}{ Male } & 28 & 33 \\
\hline & Total & 48 & 48 \\
\hline
\end{tabular}

After 12 hours of overnight fasting, a 3-ml peripheral blood samples of stroke patients were collected and centrifuged $3000 \mathrm{rpm}$ for 10 min to obtain serum (within week $1[n=48]$, week 2[n=19], week $3[n=16]$, week $4[n=18]$, week $5[n=11]$ from admission), so as to healthy controls[n=48]. All these serum samples were stored at $-80^{\circ} \mathrm{C}$ for subsequent analysis. $300 \mathrm{ul}$ of serum was mixed with $600 \mathrm{ul} 10 \%$ trichloroacetic acid, vortexed for $10 \mathrm{~s}$ and reacted for $10 \mathrm{~min}$ to remove proteins, then centrifuged $10000 \mathrm{rpm}$ for $15 \mathrm{~min}$ at 4 ${ }^{\circ} \mathrm{C}$ before experiment, and then the supernatant was used to analyze the amino acids through Amino Acid Analyzer.

\subsection{Statistical analysis}

The measured amino acid values were normally distributed, thus, differences in these parameters between two groups were assessed using the independent-samples $t$ test; certain parameter change along time in one group was assessed using one-way analysis of variance (ANOVA); comparison of count 
parameters were assessed using Chi-square test. Furthermore, receiver operating characteristic (ROC) curve was used to evaluate the diagnostic efficacy of distinguishing healthy controls and ischemic stroke patients, and the results were reported as area under the curve (AUC). All statistical analysis was performed with SPSS 25.0 (SPSS, USA) and Graphpad Prism 8(GraphPad Software, USA). Statistical significance was defined as $P<0.05$.

\section{Results}

\subsection{Different amino acid profiles between stroke patients and control subjects}

23 amino acids were identified in our study, 12 were found significantly different between the ischemic stroke patients and the healthy controls (Table 2). The average levels of phosphoserine, glutamic acid, isoleucine, leucine, phenylalanine, arginine, proline in the ischemic stroke patients were increased compared to controls, and the levels of taurine, cystine, methionine, $b$-alanine, histidine were decreased $(P<0.05)$. These results showed that there were obvious metabolic differences between two groups. To evaluate the abilities using amino acid to distinguish stroke patients and healthy controls, the results of ROC curve analyses comparing elevated and lowered amino acids levels between two groups were shown in Figure 1 and Figure 2 respectively. The AUC of P-Ser, Glu, lle, Leu, Phe, Arg, Pro in ischemic stroke patients as compared to healthy controls was $0.904,0.900,0.662,0.639,0.736,0.701,0.622$, the AUC of Tau, Cys, Met, b-Ala, His in ischemic stroke patients as compared to healthy controls was $0.773,0.970$, $0.690,0.619,0.761$, which indicated that most amino acids had high diagnostic efficiency in convalescent patients with ischemic stroke and healthy controls. 
Table 2

The average of all amino acids from healthy controls and ischemic stroke patients in the first week囚umol/L】

\begin{tabular}{|c|c|c|c|}
\hline & $\mathbf{N}$ & W1 & $P$ \\
\hline P-Ser & 13.06 & 17.16 & $<0.001$ \\
\hline Tau & 161.00 & 127.06 & $<0.001$ \\
\hline Urea & 4904.13 & 5334.34 & \\
\hline Thr & 133.53 & 137.84 & \\
\hline Ser & 172.97 & 184.28 & \\
\hline Glu & 127.28 & 219.44 & $<0.001$ \\
\hline Gly & 314.94 & 332.72 & \\
\hline Ala & 489.44 & 509.91 & \\
\hline Cit & 39.75 & 43.00 & \\
\hline a-ABA & 25.63 & 26.13 & \\
\hline Val & 274.50 & 288.34 & \\
\hline Cys & 40.44 & 12.03 & $<0.001$ \\
\hline Met & 37.84 & 32.53 & $<0.001$ \\
\hline Ile & 80.03 & 91.19 & $<0.001$ \\
\hline Leu & 162.38 & 176.34 & $<0.001$ \\
\hline Tyr & 79.72 & 75.69 & \\
\hline Phe & 102.75 & 117.50 & $<0.001$ \\
\hline b-Ala & 5.75 & 4.97 & $<0.001$ \\
\hline Om & 112.41 & 128.97 & \\
\hline Lys & 220.00 & 216.81 & \\
\hline His & 89.35 & 78.28 & $<0.001$ \\
\hline Arg & 109.66 & 128.62 & $<0.001$ \\
\hline Pro & 190.22 & 222.00 & $<0.001$ \\
\hline \multicolumn{4}{|c|}{$\begin{array}{l}\text { Abbreviations: N: normal, W1: week 1, P-Ser: Phosphoserine, Tau: Taurine, Urea: Urea, Thr: Threonine, } \\
\text { Ser: Serine, Glu: Glutamic Acid, Gly: Glycine, Ala: Alanine, Cit: Citrulline, a-ABA: a-Amino-n-butyric Acid, } \\
\text { Val: Valine, Cys: Cystine, Met: Methionine, Ile: Isoleucine, Leu: Leucine, Tyr: Tyrosine, Phe: } \\
\text { Phenylalanine, b-Ala: b-Alanine, Orn: Ornithine, Lys: Lysine, His: Histidine, Arg: Arginine, Pro: Proline }\end{array}$} \\
\hline
\end{tabular}




\subsection{Exploration of prognostic biomarkers}

The metabolic differences between convalescent patients with ischemic stroke and healthy subjects had been studied. At the same time, we monitored the trend of amino acid changes continuously and tried to seek some prognosis biomarkers for stroke patients. According to past studies, two typical amino acids (Tau, Glu) were selected and their ratio was considered as prognostic markers because that Tau can resist neurotoxicity of Glu. Tau/Glu was significant lower in stroke group in the first week of admission than control group as shown in Figure 3. Daily serum samples were obtained for 5 weeks after admission in the stroke group, and the histogram (mean) of Tau/Glu in 5 weeks was shown in Figure 4, which indicated the value of Tau/Glu increased gradually with the prognosis getting better. Due to the neuroprotective effect of Tau resists neurotoxicity of Glu, showing that Tau/Glu may be related to the prognosis and could be used as the markers for monitoring prognosis. According to the $\mathrm{BI}$ when discharged, the patients were divided into a good prognosis group $(\mathrm{B} \mid>60)$ and a poor prognosis group (BI 560$)$, and the histogram (mean) of Tau/Glu in two sub-groups were shown in Figure $5 \mathrm{a}$ and $5 \mathrm{~b}$. The value of Tau/Glu in good prognosis group elevated significantly in the 5th week comparing to 1st week, however, the value of Tau/Glu in poor prognosis group didn't have significant difference in the 5 weeks. Independent-samples t test was used to compare Tau/Glu level in two sub-groups respectively in 5 weeks from admission, and there was significant difference in the 5 th week in good and poor prognosis group showing in Figure 6. The ROC curve analyses comparing Tau/Glu between two sub-groups was shown in Figure 7, the AUC was 0.964 , and the cut-off value was 0.79 . Therefore, Tau/Glu level can be used as a prognosis marker and considering stroke patients have a good prognosis when the ratio $>0.79$.

\section{Discussion}

Ischemic stroke continues to be a serious public health problem worldwide, with a poor prognosis once it occurs. Fast diagnosis and appropriate treatment are of utmost significance to improving the outcome in patients with acute ischemic stroke or convalescent patients with ischemic stroke. Therefore, it is particularly important to investigate the metabolism and find some indicators that can evaluate the prognosis of stroke patients for the optimized care and treatment(Ormstad, Verkerk, \& Sandvik, 2016; X. Wang et al., 2020). Many studies have reported amino acid metabolism in acute ischemic stroke. In this study, we compared the amino acid profiles of convalescent patients with ischemic stroke with healthy subjects by HPLC, aiming to find the metabolic differences and prognostic markers between two groups. 24 amino acids were identified in our study, the levels of P-Ser, Glu, Ile, Leu, Phe, Arg, Pro in stroke patients increased significantly, and the levels of Tau, Cys, Met, b-Ala, His lowered in patients compared to controls, which indicated that most amino acids had high diagnostic efficiency in convalescent patients with ischemic stroke and healthy controls. Ile and Leu were reduced in acute ischemic stroke(Kimberly, Wang, Pham, Furie, \& Gerszten, 2013), but we observed that they were increased in convalescent patients in our study, which was consistent to reported research(Goulart et al., 2019). Glutamate, as a nerve excitatory amino acid, is an indicator of neural excitation and neurotoxin, and is found to be highly increased in stroke patients as studies demonstrated(Gomi et al., 2000; Meng et al., 2015). These amino 
acid metabolic differences between convalescent patients and healthy controls could be used as diagnostic markers in two groups. Given the complexity of neurobiological mechanisms of ischemic stroke, it might need a combination, rather than a single metabolite using as the diagnostic marker(D. Wang, Kong, Wu, Wang, \& Lai, 2017). So, further studies are needed.

Although advances in medicine have greatly improved the treatment of patients with ischemic stroke in recent years, there is still a high recurrence rate. Therefore, it is particularly important to find indicators that can judge the prognosis of patients. Amino acids are important nutrients in human body, the levels of free amino acids in serum could partially reflect the status of metabolic disorders, which may help to evaluate the status of patients(Xie \& Li, 2015). The stroke patients exhibited significantly higher Glu and lower Tau and Tau/Glu than the healthy controls in our study. Glu is the main excitatory neurotransmitter in the central nervous system, impaired uptake or excessive release of Glu lead to the extracellular concentration rising sharply in ischemic stroke patients, and ultimately the excitotoxicity of Glu can induce neuronal death and brain damage(Meng et al., 2015; Y. Wang et al., 2013). Ahlem Zaghmi et al demonstrated that sustained blood glutamate scavenging could enhance the protection in ischemic stroke patients(Zaghmi et al., 2020). Taurine is widely involved in neurological activities leading to neuroprotection; the inhibitory amino acid taurine is a neuromodulator, which can resist the neurotoxic effects of glutamate and mitochondrial disorders, exerting neuroprotective actions in neural tissue(Ghandforoush-Sattari, Mashayekhi, Nemati, \& Ayromlou, 2011; Morland, Pettersen, \& Hassel, 2016; Sun, Gu, Zhao, \& Xu, 2011). we monitored the trend of two amino acid changes continuously to for 5 weeks explore whether Glu, Tau or Tau/Glu can be used as prognosis biomarkers for stroke patients. According to our results, the value of Tau/Glu increased gradually with the prognosis getting better. The value of Tau/Glu in good prognosis group elevated significantly in the 5th week comparing to 1st week, however, the value of Tau/Glu in poor prognosis group didn't have significant difference in the 5 weeks, and there was significant difference in the 5 th week in good and poor prognosis group. The ROC curve comparing Tau/Glu between two sub-groups was analyzed, the AUC was 0.964 , and the cut-off value was 0.79. Therefore, Tau/Glu level can be used as a potential prognosis marker and considering convalescent stroke patients have a good prognosis when the ratio $>0.79$. Further studies are also needed to investigate the role of Tau/Glu in more patient groups as well.

\section{Conclusion}

There were obvious amino acid metabolic differences between convalescent stroke patients and healthy controls, and most amino acids had high diagnostic efficiency in two groups. The Tau/Glu level can be used as a potential prognosis marker of convalescent stroke patients. However, a limitation of this study is that the number of participants is less, we need to include more subjects to verify the potential prognosis marker. Anymore, further studies are needed to seek more biomarkers for better treatment of stroke patients.

\section{Declarations}




\section{Disclosure statement}

No potential conflict of interest was reported by the author(s).

\section{Ethics approval and consent to participate:}

The study was approved by the ethics committee in march 2019, and informed consent was obtained from all individual participants included in the study.

\section{Consent for publication:}

All the authors listed have approved for the publication of the manuscript that is enclosed.

\section{Funding:}

This research did not receive any specific grant from funding agencies in the public, commercial, or notfor-profit sectors.

\section{Data availability statement}

The data presented in this study are available upon request.

\section{Authors' contributions:}

Chenxi Zhang, Lijuan wang, Nan qiu carried out the detection of sample and data analysis, original draft preparation. Yijun shi participated in data analysis. Yaowei ding, Guoge li participated in the serum samples collection. Guanghui zheng had contributions to literatures review and final draft writing. Guojun zhang had contributions to the conception and design of the study. All authors have read and approved the final manuscript.

\section{Acknowledgements}

We wish to thank Guojun.zhang and Guanghui zheng for providing language help, writing assistance and proof reading the article etc in writing this paper.

\section{References}

1. Ghandforoush-Sattari M, Mashayekhi SO, Nemati M, Ayromlou H (2011) Changes in plasma concentration of taurine in stroke. Neurosci Lett 496(3):172-175. doi:10.1016/j.neulet.2011.04.010

2. Gomi S, Karp A, Greenberg JH (2000) Regional alterations in an excitatory amino-acid transporter, blood flow, and glucose metabolism after middle cerebral artery occlusion in the rat. Exp Brain Res 130(4):521-528. doi:10.1007/s002219900252

3. Goulart VAM, Sena MM, Mendes TO, Menezes HC, Cardeal ZL, Paiva MJN, Resende RR (2019) Amino Acid Biosignature in Plasma among Ischemic Stroke Subtypes. Biomed Res Int, 2019, 8480468. 
doi: $10.1155 / 2019 / 8480468$

4. Johnson $\mathrm{CH}$, Ivanisevic J, Siuzdak G (2016) Metabolomics: beyond biomarkers and towards mechanisms. Nat Rev Mol Cell Biol 17(7):451-459. doi:10.1038/nrm.2016.25

5. Johnson CH, Patterson AD, Idle JR, Gonzalez FJ (2012) Xenobiotic metabolomics: major impact on the metabolome. Annu Rev Pharmacol Toxicol 52:37-56. doi:10.1146/annurev-pharmtox-010611134748

6. Kimberly WT, Wang Y, Pham L, Furie KL, Gerszten RE (2013) Metabolite profiling identifies a branched chain amino acid signature in acute cardioembolic stroke. Stroke 44(5):1389-1395. doi:10.1161/STROKEAHA.111.000397

7. Meng XE, Li N, Guo DZ, Pan SY, Li H, Yang C (2015) High plasma glutamate levels are associated with poor functional outcome in acute ischemic stroke. Cell Mol Neurobiol 35(2):159-165. doi:10.1007/s10571-014-0107-0

8. Morland C, Pettersen MN, Hassel B (2016) Hyperosmolar sodium chloride is toxic to cultured neurons and causes reduction of glucose metabolism and ATP levels, an increase in glutamate uptake, and a reduction in cytosolic calcium. Neurotoxicology 54:34-43. doi:10.1016/j.neuro.2016.03.005

9. Ormstad H, Verkerk R, Sandvik L (2016) Serum Phenylalanine, Tyrosine, and their Ratio in Acute Ischemic Stroke: on the Trail of a Biomarker? J Mol Neurosci 58(1):102-108. doi:10.1007/s12031015-0659-6

10. Ramasamy DK, Dutta T, Kannan V, Chandramouleeswaran V (2021) Amino acids in post-stroke rehabilitation. Nutr Neurosci 24(6):426-431. doi:10.1080/1028415X.2019.1641295

11. Sun M, Gu Y, Zhao Y, Xu C (2011) Protective functions of taurine against experimental stroke through depressing mitochondria-mediated cell death in rats. Amino Acids 40(5):1419-1429.

doi:10.1007/s00726-010-0751-8

12. Tu WJ, Zeng XW, Deng A, Zhao SJ, Luo DZ, Ma GZ, Liu Q (2017) Circulating FABP4 (Fatty AcidBinding Protein 4) Is a Novel Prognostic Biomarker in Patients With Acute Ischemic Stroke. Stroke 48(6):1531-1538. doi:10.1161/STROKEAHA.117.017128

13. Wang D, Kong J, Wu J, Wang X, Lai M (2017) GC-MS-based metabolomics identifies an amino acid signature of acute ischemic stroke. Neurosci Lett 642:7-13. doi:10.1016/j.neulet.2017.01.039

14. Wang H, Lin J, Zheng L, Zhao J, Song B, Dai Y (2020) Texture analysis based on ADC maps and T2FLAIR images for the assessment of the severity and prognosis of ischaemic stroke. Clin Imaging 67:152-159. doi:10.1016/j.clinimag.2020.06.013

15. Wang X, Zhang L, Sun W, Pei LL, Tian M, Liang J, Song B (2020) Changes of Metabolites in Acute Ischemic Stroke and Its Subtypes. Front Neurosci 14:580929. doi:10.3389/fnins.2020.580929

16. Wang Y, Wang Y, Li M, Xu P, Gu T, Ma T, Gu S (2013) (1)H NMR-based metabolomics exploring biomarkers in rat cerebrospinal fluid after cerebral ischemia/reperfusion. Mol Biosyst 9(3):431-439. doi:10.1039/c2mb25224d

17. Xie Y, Li Z (2015) Determination of 18 Types of Amino Acids in the Serum of Ischemic Stroke Patients by High Performance Liquid Chromatography-diode Array Detector Derivatized with 6- 
Aminoquinolyl-N-hydroxysuccinimidyl Carbamate. Cell Biochem Biophys 73(1):111-115. doi:10.1007/s12013-015-0648-y

18. Zaghmi A, Dopico-Lopez A, Perez-Mato M, Iglesias-Rey R, Hervella P, Greschner AA, Gauthier MA (2020) Sustained blood glutamate scavenging enhances protection in ischemic stroke. Commun Biol 3(1):729. doi:10.1038/s42003-020-01406-1

Figures

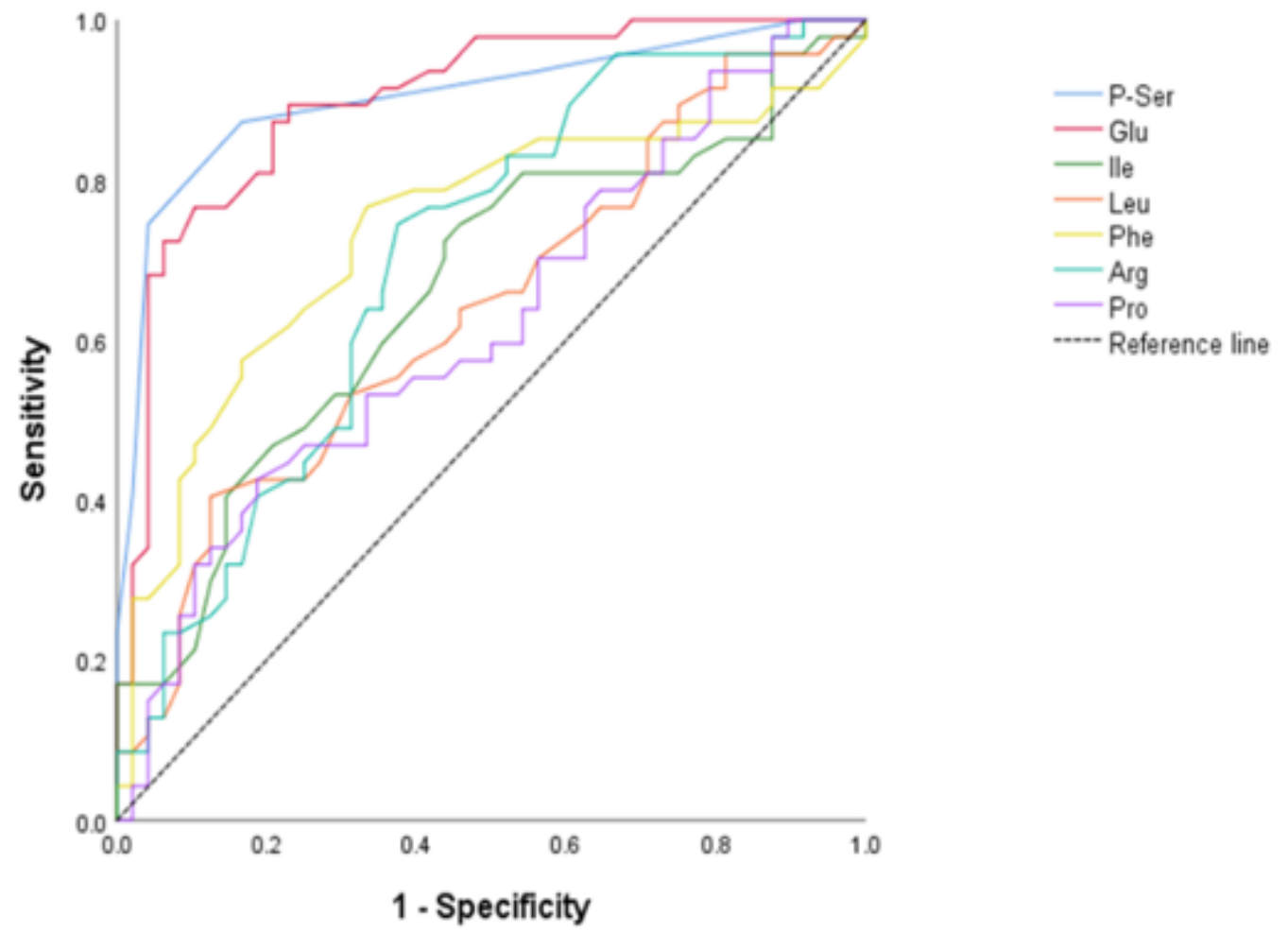

\section{Figure 1}

ROC curve analysis of the sensitivity and specificity of P-Ser, Glu, lle, Leu, Phe, Arg, Pro in ischemic stroke patients $(n=48)$ as compared to healthy controls $(n=48)$. The AUC was $0.904,0.900,0.662,0.639,0.736$, $0.701,0.622$ respectively 


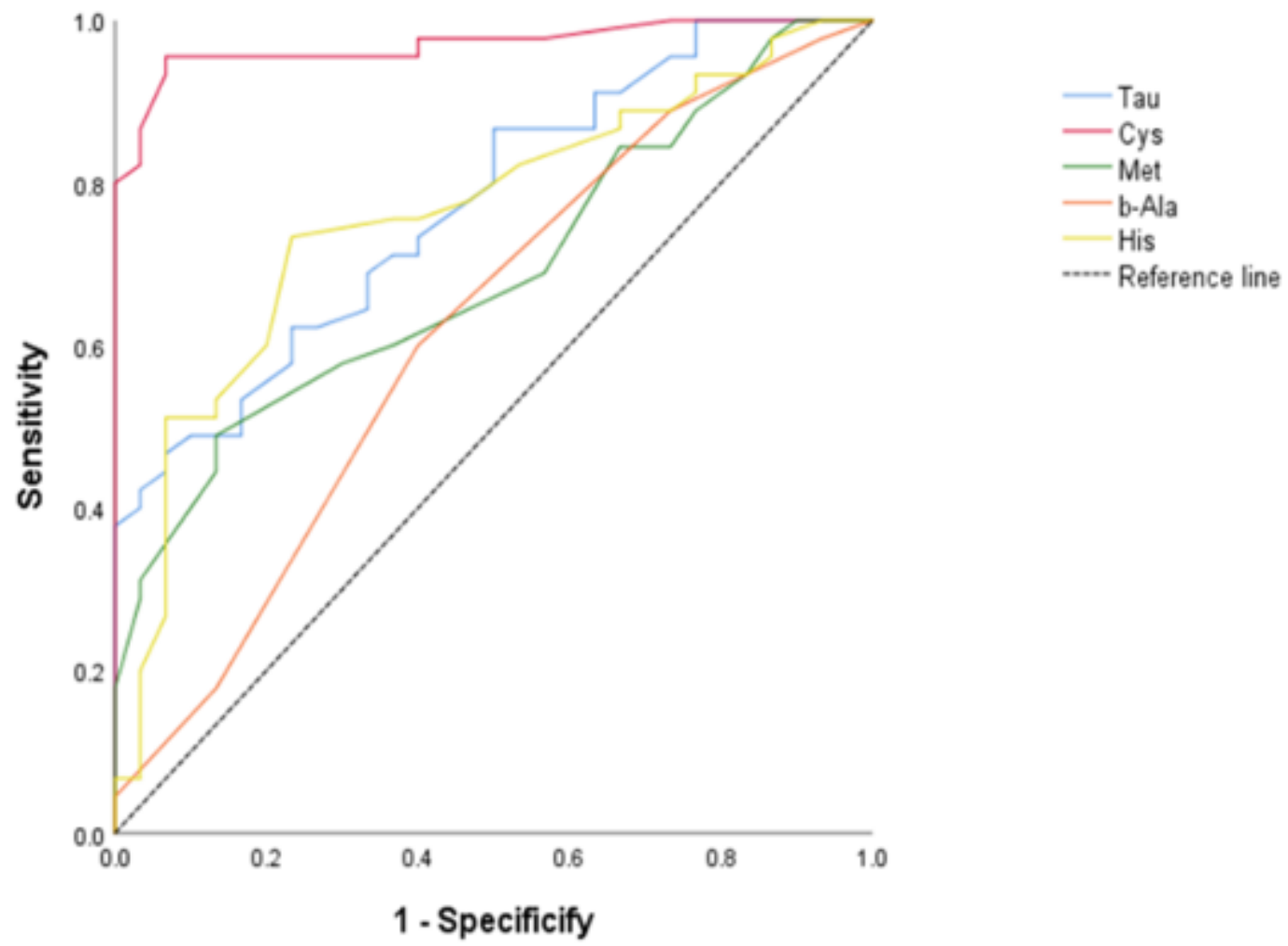

\section{Figure 2}

ROC curve analysis of the sensitivity and specificity of Tau, Cys, Met, b-Ala, His in ischemic stroke patients $(n=48)$ as compared to healthy controls $(n=48)$. The AUC was $0.773,0.970,0.690,0.619,0.761$ respectively 


\section{Tau/Glu}

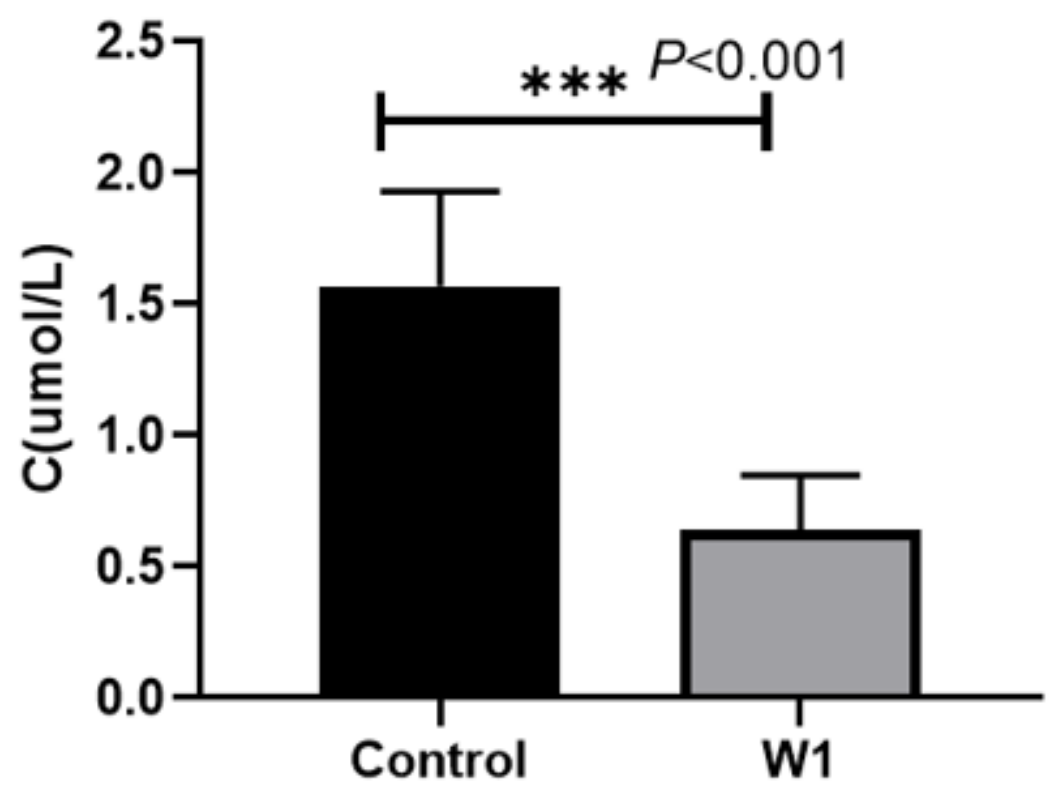

Figure 3

Histogram (mean) of Tau/Glu in stroke patients and healthy controls

\section{Tau/Glu}

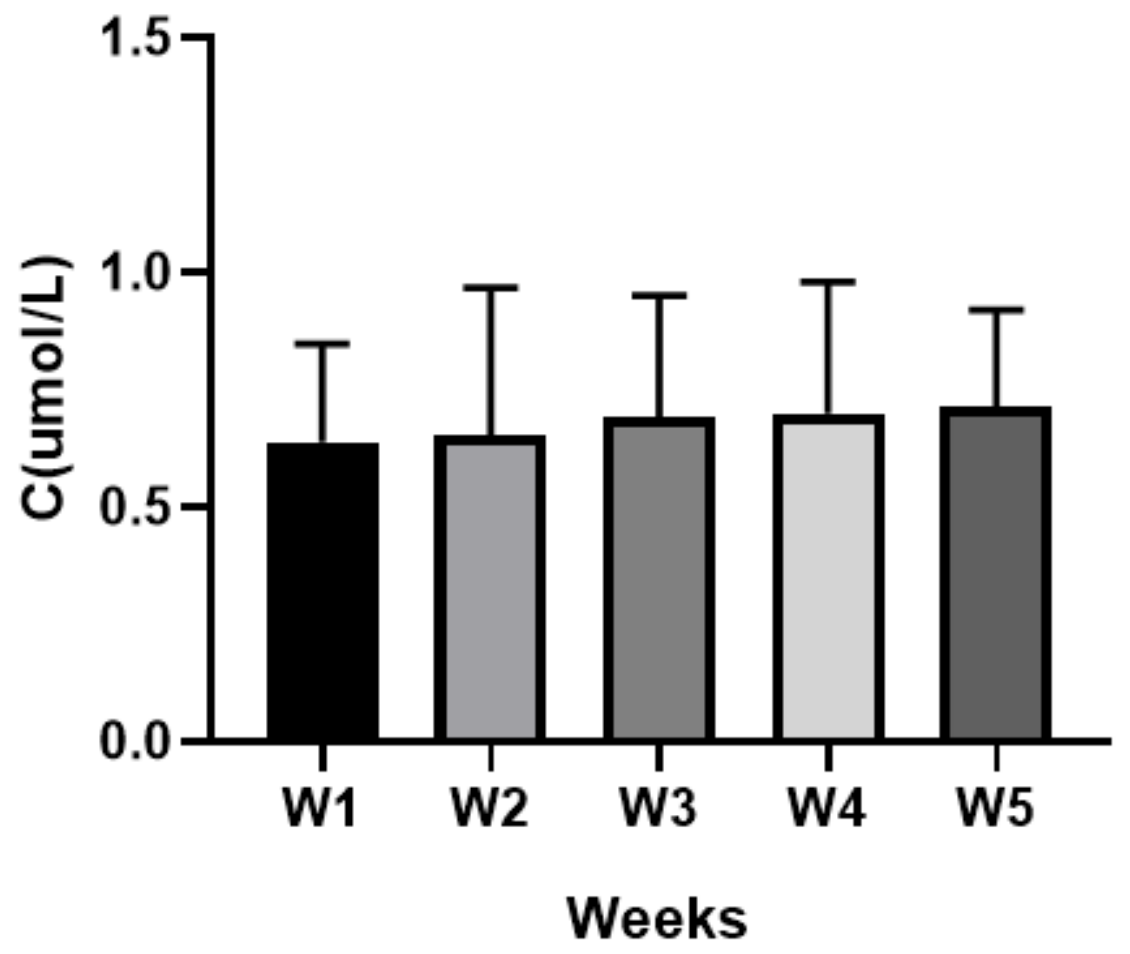


Figure 4

Histogram (mean) of Tau/Glu in the 5 weeks after admission
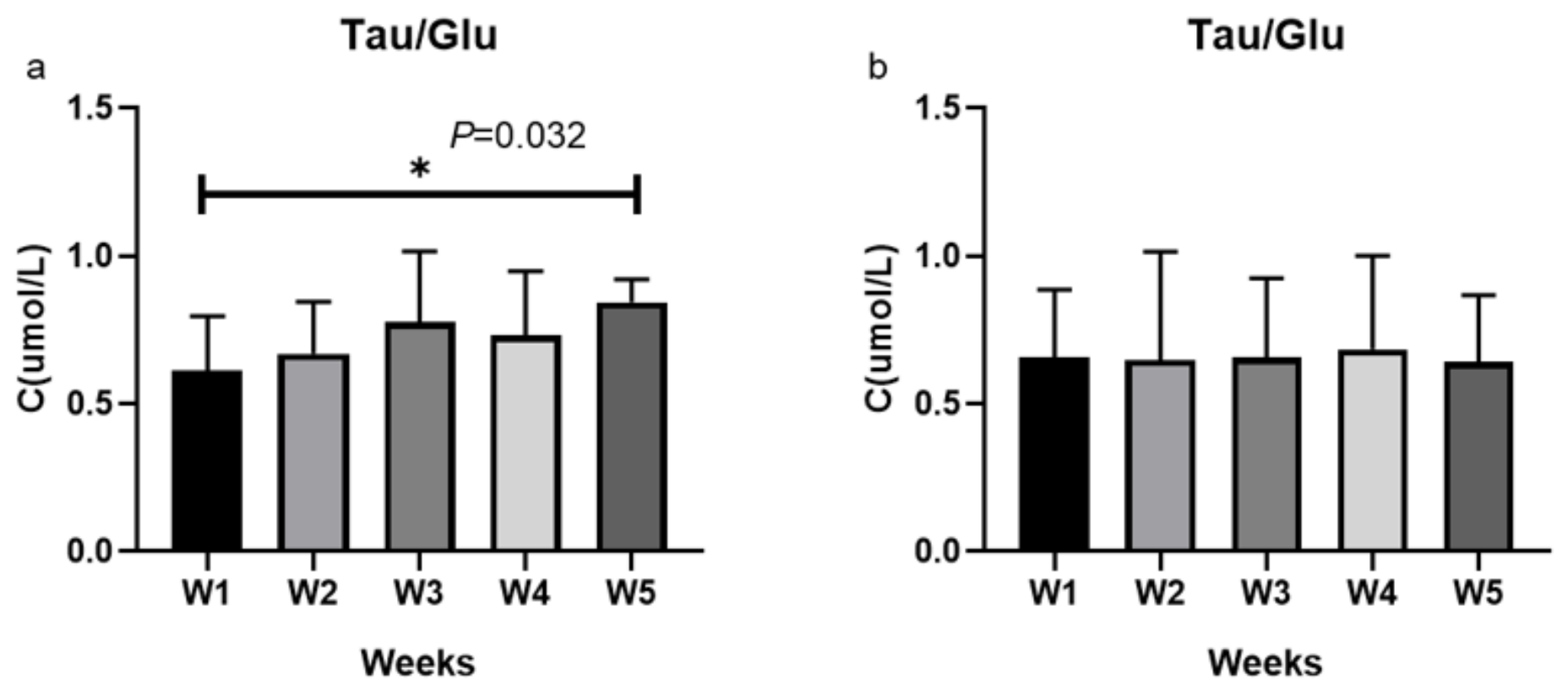

Figure 5

Histogram (mean) of Tau/Glu in the 5 weeks after admission; a:Barthel index $>608 \mathrm{~b}$ : Barthel index $\leq 60$

Tau/Glu

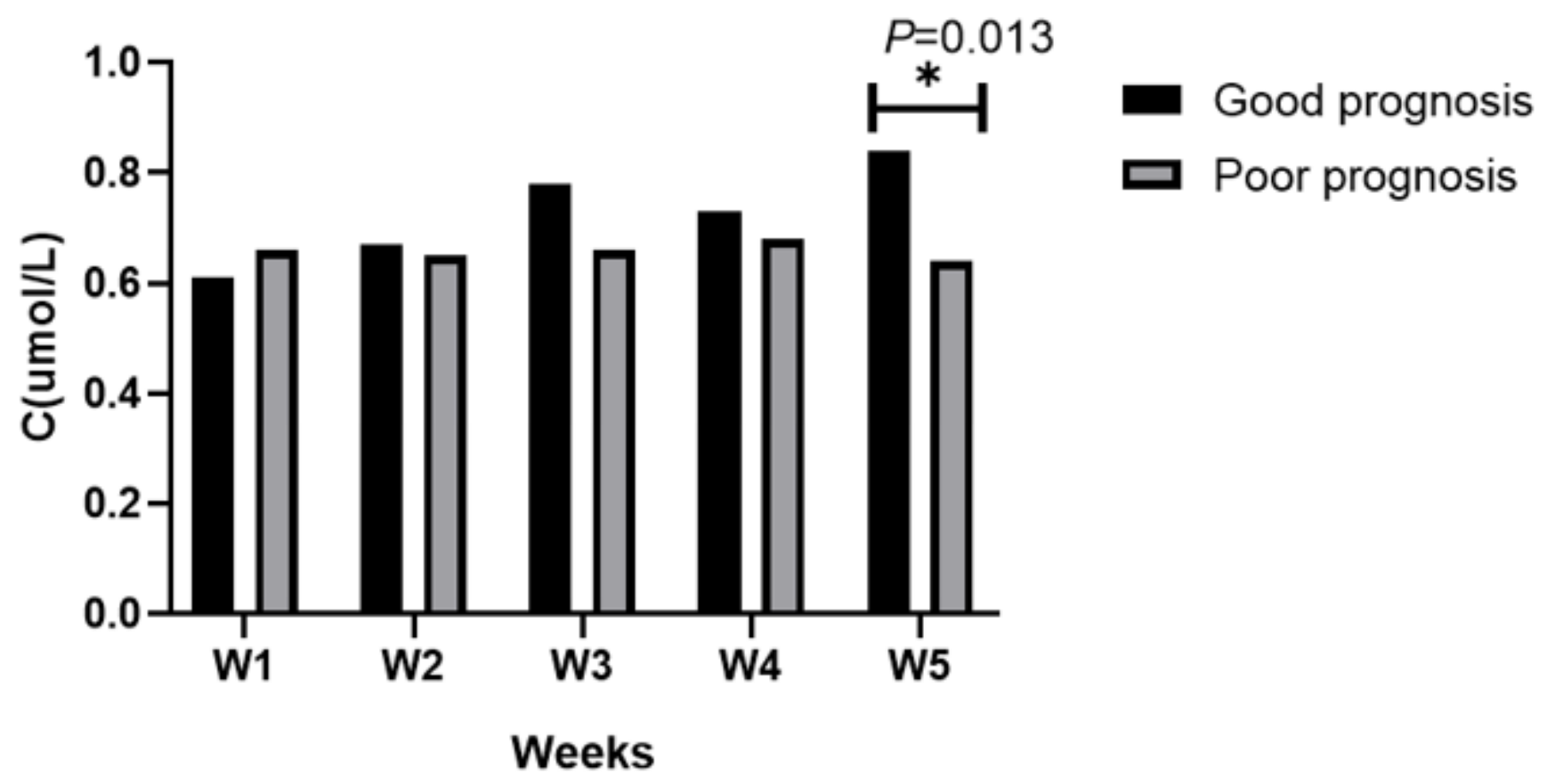

Figure 6 
Comparison of Tau/Glu level in stroke patients with good and poor prognosis

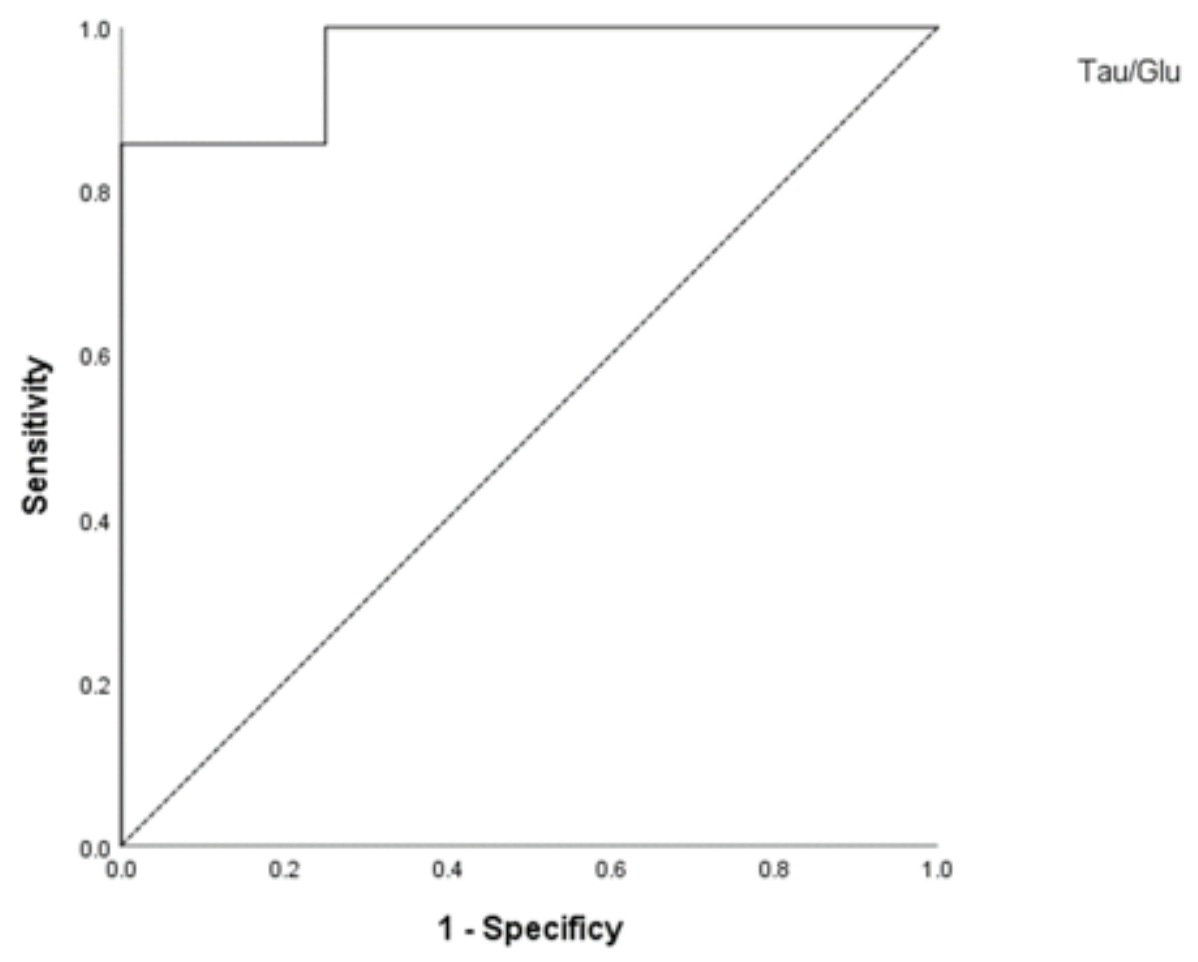

\section{Figure 7}

ROC curve of the good and the poor prognosis group in the $5^{\text {th }}$ week, the AUC was 0.964 , and the cut-off value was 0.79 